\title{
Simultaneous Curvature and Temperature Sensing Based on a Novel Mach-Zehnder Interferometer
}

\author{
Xiaoyan SUN, Haifeng DU, Xinran DONG, Youwang HU*, and Ji' an DUAN \\ State Key Laboratory of High Performance Complex Manufacturing, College of Mechanical and Electrical Engineering, \\ Central South University, Changsha 410083, China \\ *Corresponding author: Youwang HU_ E-mail:huyw@csu.edu.cn
}

\begin{abstract}
A novel fiber inline Mach-Zehnder interferometer (MZI) is proposed for simultaneous measurement of curvature and temperature. The sensor composes of single mode-multimode-dispersion compensation-multimode-single mode fiber (MMF-DCF-MMF) structure, using the direct fusion technology. The experimental results show curvature sensitivities of $-12.82 \mathrm{~nm} / \mathrm{m}^{-1}$ and $-14.42 \mathrm{~nm} / \mathrm{m}^{-1}$ in the range of $0-0.65 \mathrm{~m}^{-1}$ for two resonant dips, as well as temperature sensitivities of $57.6 \mathrm{pm} /{ }^{\circ} \mathrm{C}$ and $74.3 \mathrm{pm} /{ }^{\circ} \mathrm{C}$ within the range of $20{ }^{\circ} \mathrm{C}-150{ }^{\circ} \mathrm{C}$. In addition, the sensor has unique advantages of easy fabrication, low cost, high fringe visibility of $24 \mathrm{~dB}$, and high sensitivity, which shows a good application prospect in dual-parameters of sensing of curvature and temperature.
\end{abstract}

Keywords: Mach-Zehnder interferometer; curvature sensing; temperature sensing; simultaneous sensing

Citation: Xiaoyan SUN, Haifeng DU, Xinran DONG, Youwang HU, and Ji'an DUAN, "Simultaneous Curvature and Temperature Sensing Based on a Novel Mach-Zehnder Interferometer," Photonic Sensors, 2020, 10(2): 171-180.

\section{Introduction}

Curvature is an important physical parameter, which is used to monitor the manufacture and civil engineering, such as bridge construction and mechanical arm [1-4]. In recent decades, different types of optical fiber sensors have been widely reported due to their high sensitivity, small size, anti-electromagnetic interference, ease to be bent, and suitability for embedding in work piece for distributed measurement, such as long period fiber grating (LPFG) [5-7], fiber Bragg grating (FBG) $[8,9]$, fiber multimode interference combined with an LPFG [10], and superimposed grating [11]. However, the fabrication of the grating-based sensors is complicated and generally requires an expensive phase mask, a laser, and special optical paths for grating writing. There have been many reports on the in-fiber Mach-Zehnder interferometers (MZI) [12-14]. For example, in 2008 , V. N. Linh et al. proposed a high temperature fiber sensor with the multimode-singlemodemultimode (MMF-SMF-MMF) structure [15]. The sensor was only used for temperature sensing, and the temperature sensitivity was $88 \mathrm{pm} /{ }^{\circ} \mathrm{C}$. In 2012 , Y. Ma et al. reported a refractive index sensor based on the in-fiber MZI with the MMF-SMF-MMF structure, which had an estimated resolution of $7.1 \times 10^{-5}$ within the linear sensing range of 1.33 to 1.40 [16]. These sensors were only used for some single parameter sensing, such as temperature sensing and refractive index sensing. As optical

Received: 29 December 2018 / Revised: 7 March 2019

(C) The Author(s) 2019.This article is published with open access at Springerlink.com

DOI: $10.1007 / \mathrm{s} 13320-019-0551-\mathrm{z}$

Article type: Regular 
fiber curvature sensors, in-fiber MZIs have been attracting more and more attention due to their high sensitivity, low lost, and simple fabrication process [17-19]. Y. Gong et al. developed a fiber-optic curvature sensor based on the single mode-multimode-single mode (SMF-MMF-SMF) fiber structure [20], of which the wavelength-curvature relationship was $10.38 \mathrm{~nm} / \mathrm{m}^{-1}$ in the range of $0.25 \mathrm{~m}^{-1}-0.46 \mathrm{~m}^{-1}$. H. P. Gong et al. demonstrated an optical fiber curvature sensor based on the photonic crystal fiber (PCF) modal interferometer which was spliced between two single mode fibers (SMF), and the curvature sensitivity was $4.451 \mathrm{~nm} / \mathrm{m}^{-1}$ in the range of $0-$ $2.14 \mathrm{~m}^{-1}$ [21]. However, these structures have lower curvature sensitivities. R. H. Wang et al. proposed an in-fiber Mach-Zehnder interferometer with the MMF-SMF-MMF structure, and the curvature sensitivity was $-14.40 \mathrm{~nm} / \mathrm{m}^{-1}$ in the range of $0.08 \mathrm{~m}^{-1}-0.72 \mathrm{~m}^{-1}$ [22]. The sensor had a typical fringe extinction ratio of $20 \mathrm{~dB}$ for sensing application. Y. Zhao et al. proposed a new optical fiber curvature sensor with the MMF-TCSMF (three-core single mode fiber)-MMF structure, and the sensitivity was $-28.29 \mathrm{~nm} / \mathrm{m}^{-1}$ in the range of $2.79 \mathrm{~m}^{-1}-3.24 \mathrm{~m}^{-1}$. The sensor had an extinction ratio of around $14 \mathrm{~dB}$ [23]. These structures had higher curvature sensitivities, but the fringe visibilities were lower than that of our proposed sensor. However, the accuracy of the MZI curvature sensors designed is disturbed by the change in ambient temperature during curvature tests. It is very important to be able to distinguish these two different parameters when the sensor is applied to the detection field.

In recent years, two-parameters sensing based in-fiber MZIs have been reported. In 2012, H. Sun et al. reported an MZI-based multimode fiber-dispersion compensation fiber-multimode fiber structure for temperature and refractive index sensing. The temperature sensitivity was $118 \mathrm{pm} /{ }^{\circ} \mathrm{C}$ in the range of $20^{\circ} \mathrm{C}-250{ }^{\circ} \mathrm{C}$, and the RI sensitivity was $66.32 \mathrm{~nm} / \mathrm{RIU}$ with the sensing range of 1.33 RIU - 1.39RIU. The transmission spectrum had an extinction ratio of around $18 \mathrm{~dB}$ [24]. Y. M. Raji et al. demonstrated an abrupt tapered fiber in-line Mach-Zehnder interferometer sensor, through measurement of optical power for simultaneous sensing of temperature and curvature [25]. H. Liu et al. experimentally demonstrated an MMF-PCFMMF structure and inscribed a fiber Bragg grating (FBG) for curvature and temperature measurement. The proposed sensor had a sensitivity of $-1.03 \mathrm{~nm} / \mathrm{m}^{-1}$ at a curvature of $10 \mathrm{~m}^{-1}$ to $22.4 \mathrm{~m}^{-1}$, and the temperature sensitivity was $60.3 \mathrm{pm} /{ }^{\circ} \mathrm{C}$ in the range of $8{ }^{\circ} \mathrm{C}-100{ }^{\circ} \mathrm{C}[9]$.

In this work, we propose a novel all fiber Mach-Zehnder interferometer fiber optical sensor with the SMF-MMF-DCF(dispersion compensation fiber)-MMF-SMF (S-M-D-M-S) structure for simultaneous sensing of curvature and temperature, which was formed by splicing a piece of DCF between two MMFs, both the input and output ends of the light source are SMFs. The DCF is the main element in the sensing structure, and the MMF is used to improve the coupling efficiency at both ends of DCF. The maximum fringe visibility of the interference resonance dip exceeds $24 \mathrm{~dB}$. The experimental results show that the sensitivities of the sensor in curvature and temperature are $-14.42 \mathrm{~nm} / \mathrm{m}^{-1}$ and $74.3 \mathrm{pm} /{ }^{\circ} \mathrm{C}$, respectively. Moreover, the fabrication of the proposed sensor is very simple, low-cost, and compact in size.

\section{Sensor design and operation principle}

\subsection{Design of sensor}

The schematic of the sensor structure is shown in Fig. 1. The input and output of the sensor are all SMF (Corning SMF-28), and the sensing part is composed of MMFs (MM-S105/125-22A) and one segment of DCF (DM1010-A). The fiber core/cladding diameters of SMF, MMF, and DCF used in experiments are $9 / 125 \mu \mathrm{m}, 105 / 125 \mu \mathrm{m}$, and 
$5 / 120 \mu \mathrm{m}$, respectively. The uncoated DCF is sandwiched to MMFs, and they are spliced together directly by a commercial fusion splicer (Fujikura $80 \mathrm{~S})$. In order to obtain better interference spectra, we test different lengths of MMF and DCF in the splicing experiment. Finally, the length of DCF we choose is $30 \mathrm{~mm}$, and the length of MMF is $5 \mathrm{~mm}$ in experiments. The MZI sensor has a total of four fusion regions, and the total splicing loss is about $2 \mathrm{~dB}$. A light source $(\mathrm{C}+\mathrm{L}$, Shanghai Hanyu) with a wavelength range of $1528 \mathrm{~nm}-1602 \mathrm{~nm}$ is launched into the MZI structure. The output spectrum is detected with an optical spectrum analyzer (OSA, Agilent 86142B, 600 - $1700 \mathrm{~nm}$ ).

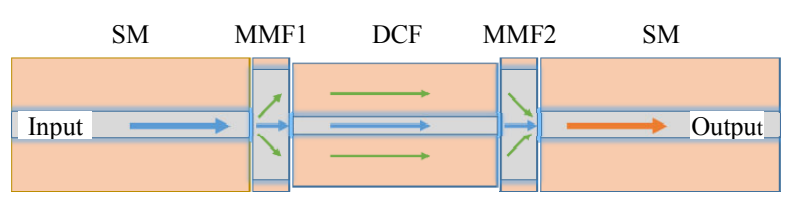

Fig. 1 Schematic of the MMF-DCF-MMF structure.

\subsection{Principle of the sensor}

The two MMF sections play the roles of core and cladding modes coupling and recoupling, and the DCF section performs as the interference area. In order to study the MZI interference principle, a tunable laser is used as the light source, and an infrared camera is used to capture the output patterns at the output end face of different fiber splicing structures. Figure 2 shows the intensity distributions of the core and the cladding modes, and the intensity distribution can be collected by using the Micron Viewer (MODEL 7290A). When the light propagates from input SMF into MMF1, the effective area of the mode field is expanded evidently because of the large diameter of MMF, as shown in Figs. 2(a) and 2(b). Then, a part of the light is coupled into the cladding modes of the DCF from the MMF1-DCF fusion region due to the mode field mismatch, as shown in Fig. 2(c). In the DCF-MMF2 fusion region, the light of the DCF cladding mode will be coupled with the MMF2 core, and the area of mode distribution reduces, which is shown in
Fig. 2(d). Finally, they are coupled with the fundamental mode of the output SMF and form an interference device.
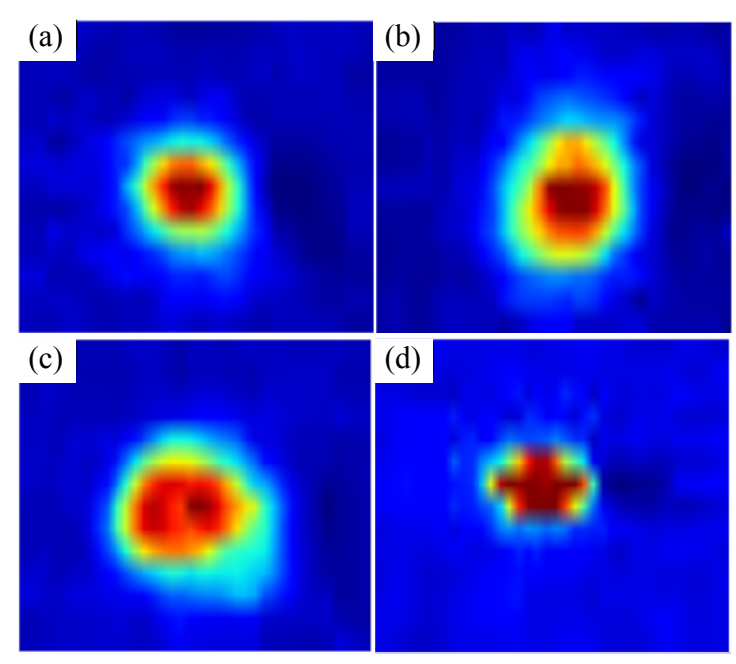

Fig. 2 Output patterns of different fiber splicing structures: (a) SMF, (b) SMF-MMF, (c) SMF-MMF-DCF, and (d) SMF-MMF-DCF-MMF-SMF.

Theoretically, the transmitted light intensity of the MZI can be expressed by the classic two-beam interference equation:

$$
I=I_{1}+I_{2}+2 \sqrt{I_{1} I_{2}} \cos \phi_{m}
$$

where $I$ is the intensity of the interference signal; $I_{1}$ and $I_{2}$ are the intensities of the light propagating along the fiber core and cladding, respectively. The phase difference $\left(\phi_{m}\right)$ between the cladding mode and core mode after propagating through DCF can be written as

$$
\phi_{m}=\frac{2 \pi\left(n_{\mathrm{eff}}^{\mathrm{core}}-n_{\mathrm{eff}}^{\mathrm{clad}}\right)}{\lambda} L=\frac{2 \pi \Delta n_{\mathrm{eff}}}{\lambda} L
$$

where $\lambda$ is the dip wavelength of the interference spectrum; $L$ is the interferometer length; $n_{\text {eff }}^{\text {core }}, n_{\text {eff }}^{\text {clad }}$, and $\Delta n_{\text {eff }}$ are the effective indexes of core mode and cladding mode, and the effective refractive index difference between the core mode and cladding mode, respectively.

According to (1), the free spectral range (FSR) between two interference dips can be expressed as

$$
F S R \approx \frac{\lambda^{2}}{\Delta n_{\mathrm{eff}} L} .
$$

According to (3), we can see that the FSR 
between two interference dips is inverse to the length of the DCF. The longer $L$ is, the smaller of $F S R$ is, and the more interference dips can be observed in the measurement range.

Figure 3 shows the schematic of the bending DCF fiber, when the DCF is subjected to external bending, the inner part of the fiber (negative $x$-direction) is compressed, and the outer part (positive $x$-direction) is stretched. The refractive index distribution across the fiber cross section can be present as

$$
n=n_{0}\left(1+\frac{x}{R}\right)
$$

where $m_{0}, R$, and $x$ are the refractive index profile when the fiber is straight, the curvature radius, and a coordinate along a line connecting the center of fiber and the center of curvature, respectively. According to (4), the refractive index of the compressed inner part becomes lower, and that of the stretched outer part becomes higher when the DCF is bent.

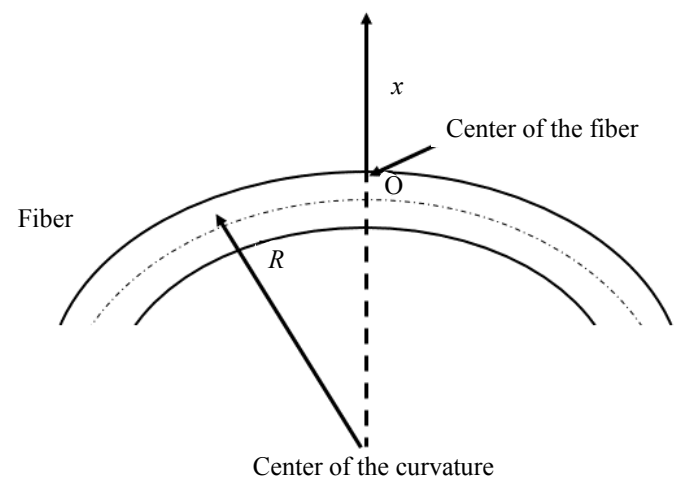

Fig. 3 Diagram of bending fiber.

Due to different thermo-optical coefficients and thermal expansion coefficients of the core and cladding modes, the effective refractive indexes of core and cladding modes change differently when the ambient temperature changes. Thus, the changes in $\Delta n_{\text {eff }}$ can lead to the interference dips shift in the transmission spectrum. Therefore, the temperature sensing information can be expressed by demodulating the resonance wavelength variation. Combining (2), the resonance wavelength $(\lambda)$ can be expressed as

$$
\lambda=\frac{2 \pi L}{(2 k+1) \pi} \Delta n_{\mathrm{eff}}
$$

where $k$ is an integer. Therefore, when the ambient temperature changes, the resonance wavelength shift $(\delta \lambda)$ can be calculated as

$$
\frac{\delta \lambda}{\delta T}=\frac{2 \pi L}{(2 k+1) \pi} \frac{\delta n_{\text {eff }}}{\delta T}+\frac{2 \pi \Delta n_{\text {eff }}}{(2 k+1) \pi} \frac{\delta L}{\delta T}
$$

where $\delta n_{\text {eff }}$ is the variation in effective refractive index difference. From (6), we can see the resonance wavelength shift $(\delta \lambda)$ is related to the length of the DCF, thermo-optical coefficient, and thermal expansion coefficient of the DCF.

\subsection{Experimental measurement method}

The experimental setup for the curvature measurement is shown in Fig. 4. The broadband light emits from a light source to the sensing structure, and then the interference spectrum is recorded by an optical spectrum analyzer. The two ends of the sensor are two masses that are used to keep the fiber straight. When we do the bending tests, the sensor is attached to a steel ruler. We can realize the accurate bending of the fiber by controlling the bending of steel ruler using the micrometer screw. The steel ruler can keep the sensor on its axis when the bending is applied. The length of the steel ruler used in the bending part is $180 \mathrm{~mm}$, and the S-M-D-M-S structure in the middle is located in the center of two fixed stations. The S-M-D-M-S structure can bend freely and synchronously to the metal beam. The curvature of the sensing structure can be determined as

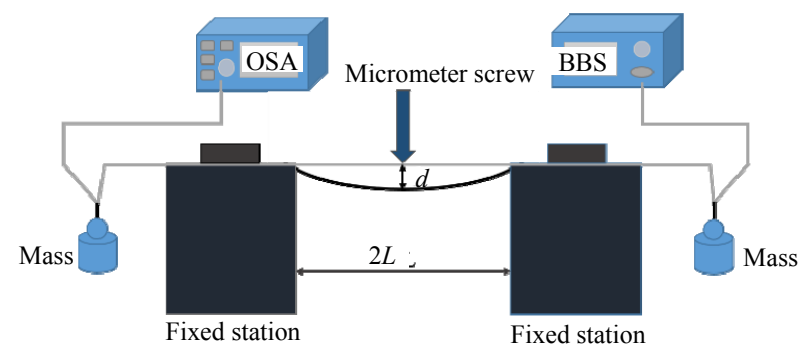

Fig. 4 Experimental setup diagram of the MZI sensor for curvature measurement. 


$$
C=\frac{1}{R}=\frac{2 d}{d^{2}+L^{2}}
$$

where $C$ is the curvature, $R$ is the curvature radius, $L$ is the half distance between the edges of the two fixed stations, and $d$ is the bending displacement adjusted by a micrometer screw.

\section{Experimental results and discussion}

To verify the effectiveness of the inserted MMF, two different kinds of MZI are fabricated: one is the SMF-DCF-SMF structure, and the other is the SMF-MMF-DCF-MMF-SMF structure. In the experiment, the lengths of the MMF and DCF are about $5 \mathrm{~mm}$ and $30 \mathrm{~mm}$ to obtain a good interference pattern, and the transmission spectra are given in Fig. 5. When the sensor is the SMF-DCF-SMF structure, there are many peaks in the transmitted interference spectrum as shown in Fig. 5(a).

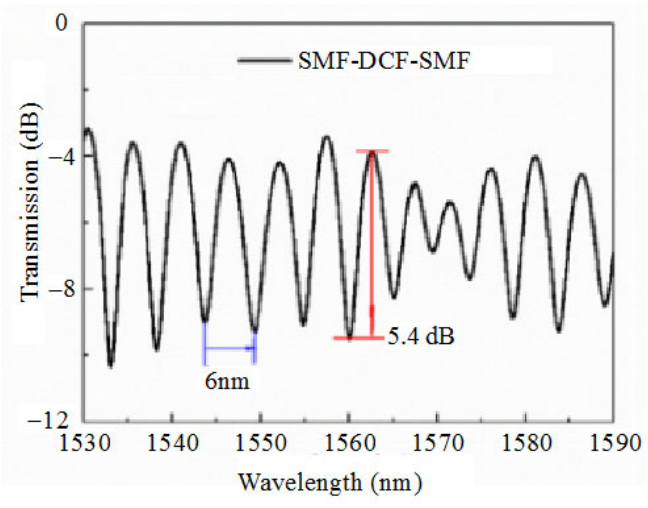

(a)
However, these peaks have low transmission loss (only $5.4 \mathrm{~dB}$ ), and it is difficult to detect. We can see from Fig. 5(b) that when the sensor is the SMF-MMF-DCF-MMF-SMF structure, the transmission spectra show two obvious interference peaks, namely Dip $1(1544.48 \mathrm{~nm})$ and Dip 2 $(1565.92 \mathrm{~nm})$, and Dip 2 has a higher transmission loss of $24 \mathrm{~dB}$. The result shows that the MMF can improve the coupling efficiency in order to illustrate the role of the MMF in the proposed sensor and its influence on bending sensing. We do the curvature measurement of the SMF-DCF-SMF structure and choose one of the peaks to illustrate the curvature sensitivity, and the results are shown in Fig. 6. According to Fig. 6, the curvature sensitivity of the SMF-DCF-SMF structure is $-1.52 \mathrm{~nm} / \mathrm{m}^{-1}$ in the range of $0-0.65 \mathrm{~m}^{-1}$, and the linear fitting is 0.9681 .

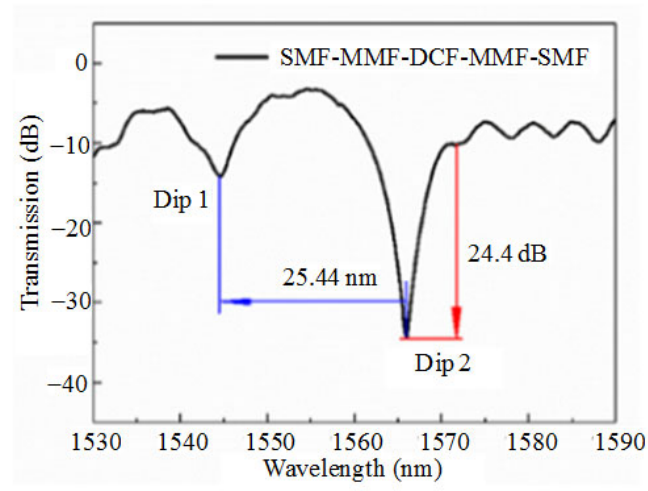

(b)

Fig. 5 Interference spectra for two different MZI structures: (a) SMF-DCF-MMF and (b) SMF-MMF-DCF-MMF-SMF.

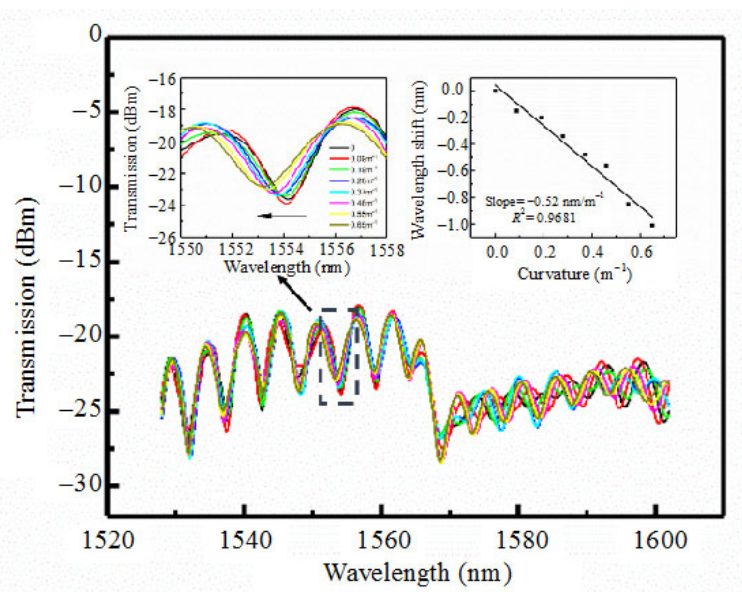

Fig. 6 Transmission spectrum change of the SMF-DCF-SMF structure.

Figure 7 shows the transmission spectra change of the MZI as the applied curvature increases. It can be seen that the interference fringe shifts toward a shorter wavelength direction when the curvature is increased gradually from 0 to $0.65 \mathrm{~m}^{-1}$. The total wavelength shift variation in Dips 1 and 2 are $8.01 \mathrm{~nm}$ and $9.48 \mathrm{~nm}$, respectively, as shown in Figs. $7(\mathrm{a})$ and $7(\mathrm{c})$. The linear fittings of the wavelength shift and curvature are illustrated in Figs. 7(b) and 7(d). The curvature sensitivities for Dip 1 and Dip2 are $-12.82 \mathrm{~nm} / \mathrm{m}^{-1}$ and $-14.42 \mathrm{~nm} / \mathrm{m}^{-1}$, with the linear regression value $R^{2}$ being 0.9894 and 0.9934 , respectively. Compared with the SMF-DCF-SMF, 
the SMF-MMF-DCF-MMF-SMF has a high curvature sensitivity. In order to analyze the mode changes and power distribution of the interference modes with an increase in the curvature, the transmission spectra in Fig. 7 are analyzed by Fourier transform [26] to get the spatial frequency spectra, and the frequency spectra of two curvatures $\left(0\right.$ and $\left.0.65 \mathrm{~m}^{-1}\right)$ are shown in Fig. 8. It can be seen that the dominant intensity peak at zero relates to the

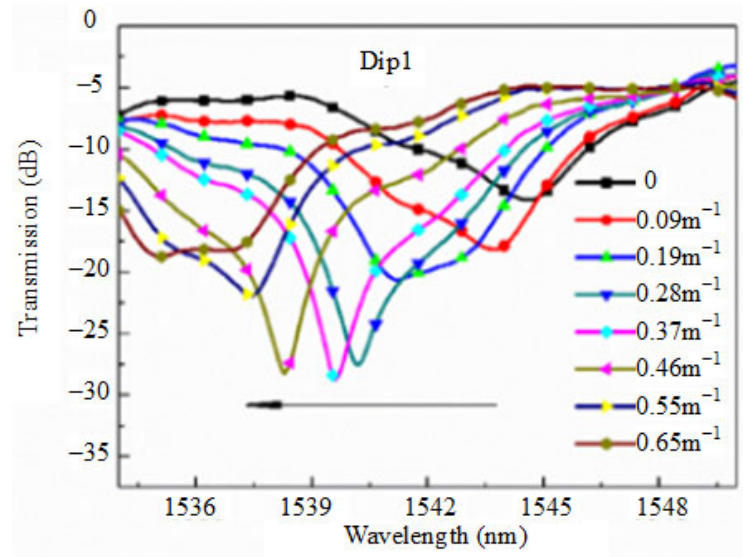

(a)

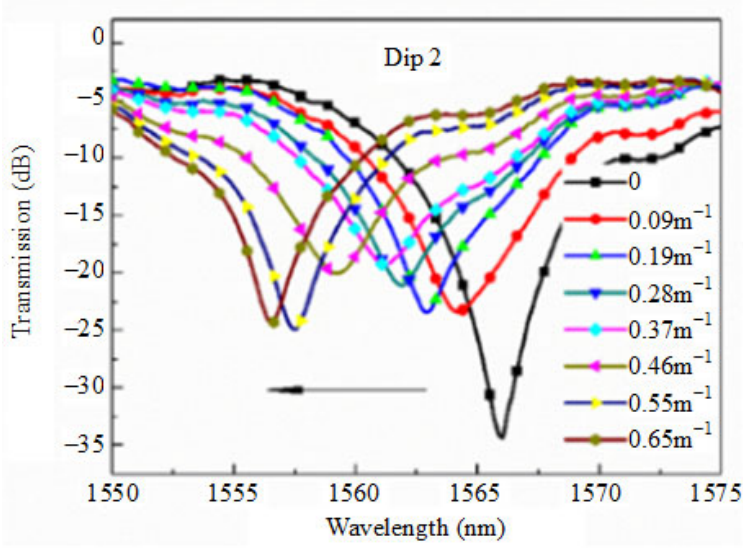

(c) core modes and the intensity mode coupling of different lengths mainly occurs between the core mode and the low-order cladding mode. There is one dominantly excited cladding mode for the MZI before bending $\left(0 \mathrm{~m}^{-1}\right)$ and one after bending $\left(0.65 \mathrm{~m}^{-1}\right)$ locating at $0.0538 \mathrm{~nm}^{-1}$ and $0.0406 \mathrm{~nm}^{-1}$, respectively. This means that the order of dominant cladding modes excited in the MZI with curvature increasing is lower than that without bending.

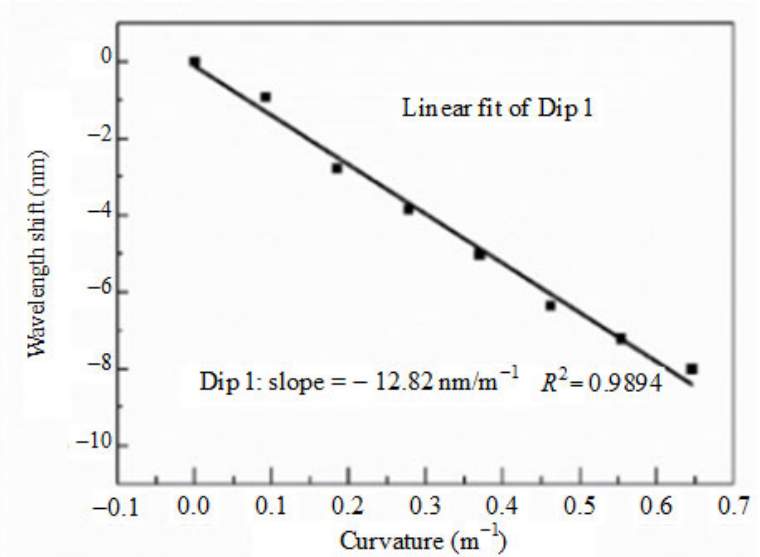

(b)

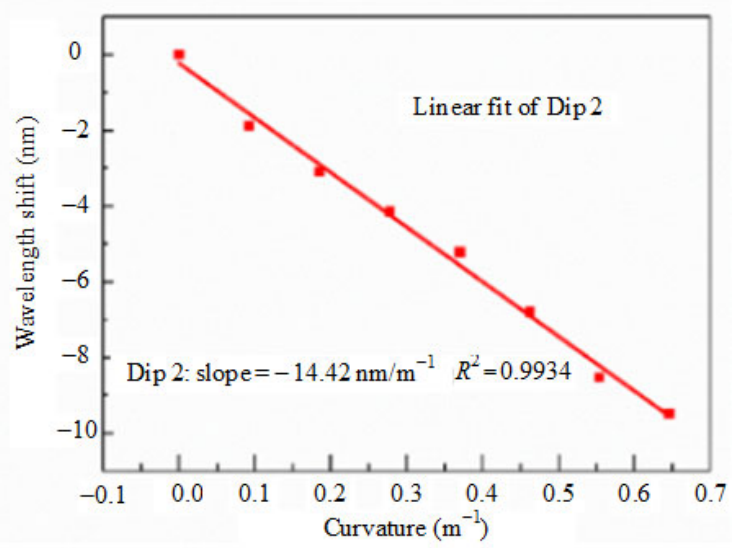

(d)

Fig. 7 Results of the curvature response experiment: (a) and (c) transmission spectrum change of the MZI as the curvature increases; (b) and (d) linear relationship between the curvature and the wavelength shift.

In the experiment, the temperature response of the SMF-MMF-DCF-MMF-SMF structure is also investigated, and the transmission spectra shift with the temperature change is shown in Fig. 9. It can be seen that the resonant wavelength shifts to the longer wavelength with the temperature increasing from $20{ }^{\circ} \mathrm{C}$ to $150{ }^{\circ} \mathrm{C}$, as shown in Figs. 9 (a) and 9(c).
The total wavelength shift variations in Dips 1 and 2 are $7.62 \mathrm{~nm}$ and $9.75 \mathrm{~nm}$, respectively. The linear relationships between the temperature and the wavelength shift are shown in Figs.9 (b) and 9(d). The temperature sensitivities of Dips 1 and 2 are $0.0576 \mathrm{~nm} /{ }^{\circ} \mathrm{C}$ and $0.0743 \mathrm{~nm} /{ }^{\circ} \mathrm{C}$ in the range from $20{ }^{\circ} \mathrm{C}$ to $150{ }^{\circ} \mathrm{C}$, and the linear 
regression values are 0.9854 and 0.9779 , respectively.

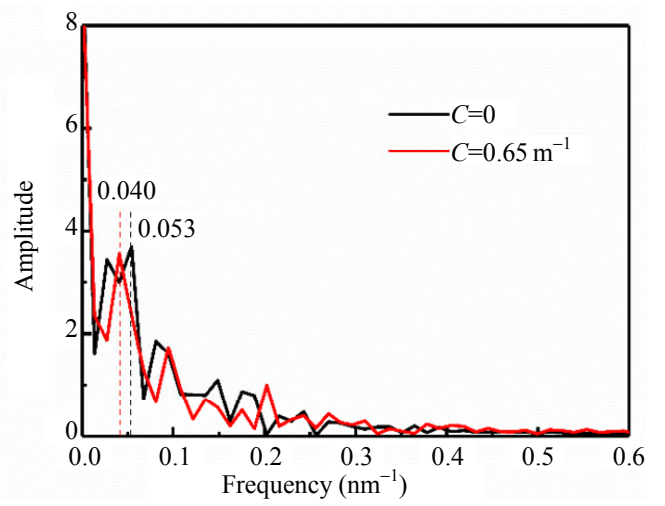

Fig. 8 Spatial frequency of the MZI before bending $\left(0 \mathrm{~m}^{-1}\right)$ and after bending $\left(0.65 \mathrm{~m}^{-1}\right)$.

A comparison between the proposed MZI and

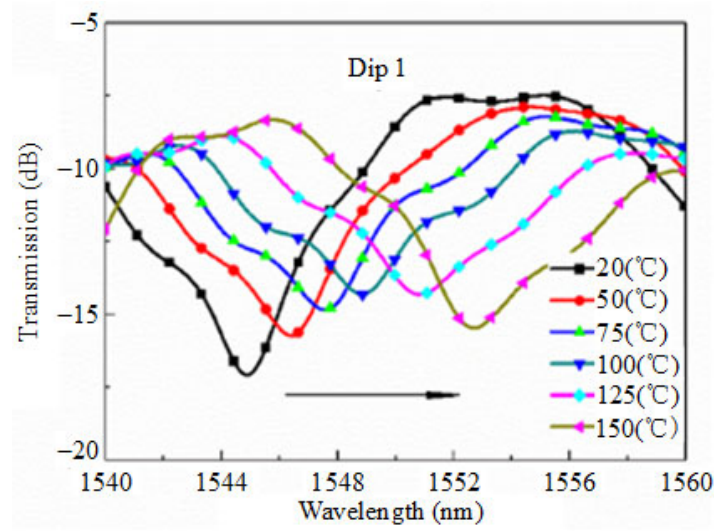

(a)

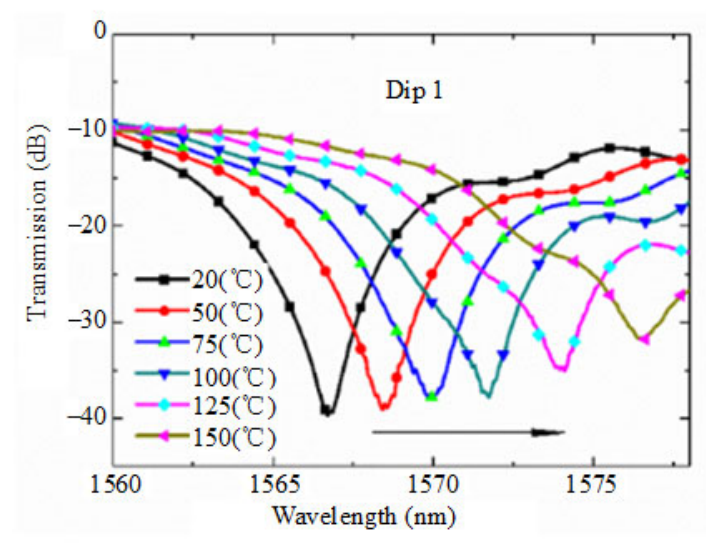

(c) the related sensors is listed in Table 1 . Table 1 shows that the sensitivities of the proposed sensor have higher curvature sensitivity and temperature sensitivity than those of the PCF-MZI and FBG $\left(4.06 \mathrm{~nm} / \mathrm{m}^{-1}\right.$ and $\left.6.3 \mathrm{pm} /{ }^{\circ} \mathrm{C}\right) \quad$ [8], and MMF-PCF-MMF inscribed FBG $\left(-1.36 \mathrm{~nm} / \mathrm{m}^{-1}\right.$ and $60.3 \mathrm{pm} /{ }^{\circ} \mathrm{C}$ ) [9]. However, the sensor we report has lower curvature sensitivity than the four-core fiber multipath MZI (20.18 nm/ $\left.\mathrm{m}^{-1}\right)$ [28], MMFTCF(twin core fiber)-MMF $\left(103.35 \mathrm{~nm} / \mathrm{m}^{-1}\right)$ [30], and MMF-TCSMF-MMF $\left(-28.29 \mathrm{~nm} / \mathrm{m}^{-1}\right)$ [31], respectively, because TCSMF, TCF, and four-core fiber are a kind of specialty optical fibers with the multi-core structure separately containing only one core mode.

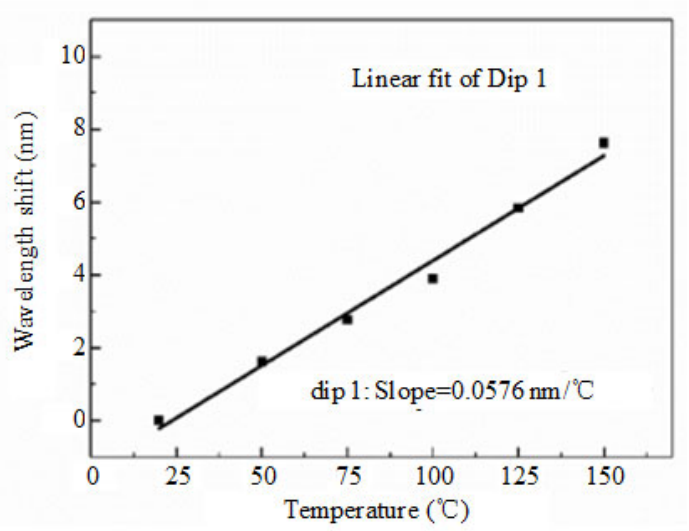

(b)

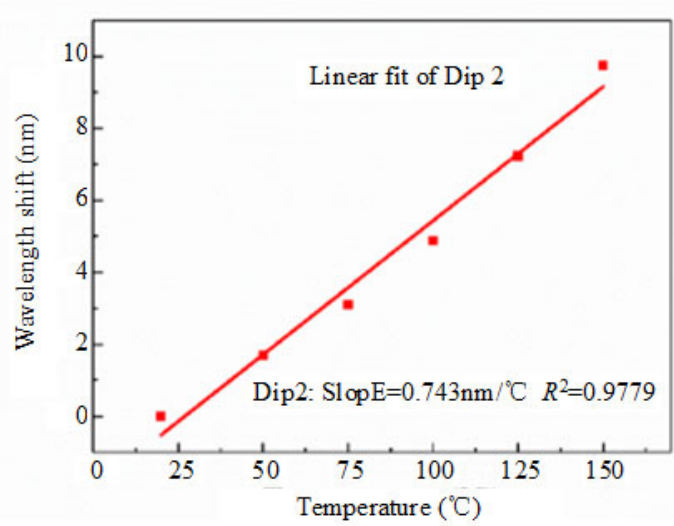

(d)

Fig. 9 Results of temperature response experiment: (a) and (c) transmission spectrum change of the MZI as the temperature increases; (b) and (d) the linear relationship between the temperature and the wavelength shift. 
Table 1 Comparison between the proposed sensor and other MZIs.

\begin{tabular}{cccccc}
\hline Sensor structure & $\begin{array}{c}\text { Curvature sensitivity } \\
\left(\mathrm{nm} / \mathrm{m}^{-1}\right)\end{array}$ & $\begin{array}{c}\text { Curvature ranges } \\
\left(\mathrm{m}^{-1}\right)\end{array}$ & $\begin{array}{c}\text { Temperature sensitivity } \\
\left(\mathrm{pm} /{ }^{\circ} \mathrm{C}\right)\end{array}$ & $\begin{array}{c}\text { Temperature range } \\
\left({ }^{\circ} \mathrm{C}\right)\end{array}$ & Reference \\
\hline Cascaded fiber interferometer & 4.362 & $0-1.134$ & 108 & $20-60$ & {$[27]$} \\
PCF-MZI and FBG & 4.06 & $0-3$ & 6.3 & $25-100$ & {$[8]$} \\
Four-core fiber multipath MZI & 20.18 & $0.3305-0.5481$ & 209 & $0-50$ & {$[28]$} \\
SMF with core-offset splicing & -22.947 & $0.35312-2.8127$ & 77.6 & $20-80$ & {$[29]$} \\
MMF-PCF-MMF with FBG & -1.03 & $10-22.4$ & 60.3 & $4-100$ & {$[9]$} \\
MMF-TCF-MMF & 103.35 & $0.24-0.6$ & 431 & $20-70$ & {$[30]$} \\
MMF-TCSMF-MMF & -28.29 & $2.79-3.24$ & 2.2 & $20-73$ & {$[31]$} \\
This work & -14.42 & $0-0.65$ & 74.3 & $20-150$ & \\
\hline
\end{tabular}

The curvature and temperature resolutions of the sensor can be obtained by using the error analysis method when the resolution $\delta(\Delta \mathrm{Dip})$ of the OSA is known, and the curvature and temperature resolutions of the sensor can be represented by [32]

$$
\left[\begin{array}{l}
\delta(\Delta C) \\
\delta(\Delta T)
\end{array}\right]= \pm\left[\begin{array}{ll}
\left|K_{\text {Dip } 2, T}\right| & \left|K_{\text {Dip } 1, T}\right| \\
\left|K_{\text {Dip } 2, C}\right| & \left|K_{\text {Dip }, C}\right|
\end{array}\right]\left[\begin{array}{l}
\delta(\Delta \text { Dip } 1) \\
\delta(\Delta \text { Dip } 2)
\end{array}\right] .
$$

In the experiment, the OSA has a wavelength measurement resolution of $10 \mathrm{pm}$. According to (12), the theoretical curvature and temperature resolutions of the proposed sensor are $\pm 0.01 \mathrm{~m}^{-1}$ and $\pm 2.23{ }^{\circ} \mathrm{C}$, respectively.

\section{Conclusions}

In this paper, we propose and experimentally demonstrate a novel optical fiber Mach-Zehnder interferometer with the S-M-D-M-S structure, which is used to simultaneously measure the curvature and temperature. By applying the coupled-mode theory, we theoretically analyze the interference principle of the sensor. The extinction ratio of the MZI is up to $24 \mathrm{~dB}$. The curvature sensitivity of the sensor is $-14.42 \mathrm{~nm} / \mathrm{m}^{-1}$ in the range of $0-0.65 \mathrm{~m}^{-1}$, and the temperature sensitivity is $74.3 \mathrm{pm} /{ }^{\circ} \mathrm{C}$ in the range of $20{ }^{\circ} \mathrm{C}-150{ }^{\circ} \mathrm{C}$ in the experiment. The experimental result shows that the sensor is suited for curvature and temperature simultaneous measurement. In addition, the novel sensor is compact, easy to fabrication, and highly sensitive, and it has many great potential applications, such as in bridge construction and mechanical arm detections.

\section{Acknowledgement}

This research work is supported by the National Natural Science Foundation of China (NSFC) (Grant Nos. 51875584 and 51875585), the National Key Research and Development Program of China (Grant Nos. 2017YFB1104800 and 2018YFB1107803), and the Fundamental Research Funds for the Central Universities of Central South University (Grant No. 1053320170003).

Open Access This article is distributed under the terms of the Creative Commons Attribution 4.0 International License (http://creativecommons.org/licenses/by/4.0/), which permits unrestricted use, distribution, and reproduction in any medium, provided you give appropriate credit to the original author(s) and the source, provide a link to the Creative Commons license, and indicate if changes were made.

\section{References}

[1] H. Song, H. Gong, K. Ni, and X. Dong, "All fiber curvature sensor based on modal interferometer with waist enlarge splicing," Sensor and Actuators A: Physical, 2013, 203: 103-106.

[2] J. Wo, Q. Sun, X. Li, D. Liu, and P. P. Shum, "Biconical-taper-assisted fiber interferometer with modes coupling enhancement for high-sensitive curvature measurement," Applied Physics B: Lasers, 2014, 115: 1-8.

[3] Y. Zhao, L. Cai, and X. G. Li, "In-fiber modal interferometer for simultaneous measurement of curvature and temperature based on hollow core fiber," Optics and Laser Technology, 2017, 92: 
138-141.

[4] G. Fu, Y. Li, X. Fu, W. Jin, and W. Bi, "Temperature insensitive curvature sensor based on cascading photonic crystal fiber," Optical Fiber Technology, 2018, 41: 64-68.

[5] M. Xiong, H. Gong, Z. Wang, C. L. Zhao, and X. Dong, "Fiber curvature sensor based on spherical-shape structures and long-period grating," Optics and Lasers in Engineering, 2016, 86: 356-359.

[6] C. Luo, P. Lu, J. Chen, S. Wang, C. Zhang, and X. $\mathrm{Fu}$, "All-fiber curvature sensor based on offset splicing structure cascaded with long-period fiber grating," in Proceeding 14th International Conference on Optical Communications and Networks, Nanjing, China, 2015, pp. 1-3.

[7] X. R. Dong, Z. Xie, Y. X. Song, K. Yin, D. K. Chu, and J. A. Duan, "High temperature-sensitivity sensor based on long period fiber grating inscribed with femtosecond laser transversal-scanning method," Chinese Optics Letters, 2017, 15(9): 51-55.

[8] Y. Zhou, W. Zhou, C. C. Chan, C. W. Wei, L. Y. Shao, J. Cheng, et al., "Simultaneous measurement of curvature and temperature based on PCF-based interferometer and fiber Bragg grating," Optics Communications, 2011, 284(24): 5669-5672.

[9] L. Hui, H. Yang, X. Qiao, Y. Wang, X. Liu, Y. S. Lee, et al., "Curvature and temperature measurement based on a few-mode PCF formed M-Z-I and an embedded FBG," Sensors, 2017, 17(8): 1725-1735.

[10] O. Frazão, J. Viegas, P. Caldas, J. L. Santos, F. M. Araújo, L. A. Ferreira, et al., "All-fiber Mach-Zehnder curvature sensor based on multimode interference combined with a long-period grating," Optics Letters, 2007, 32(21): 3074-3076.

[11] X. Dong, Y. Liu, L. Y. Shao, J. Kang, and C. L. Zhao, "Temperature-independent fiber bending sensor based on a superimposed grating," IEEE Sensors Journal, 2011, 11(11): 3019-3022.

[12] X. R. Dong, H. F. Du, Z. Luo, K. Yin, and J. A. Duan, "Highly sensitive refractive index sensor based on novel Mach-Zehnder interferometer with multimode fiber-thin core fiber-multimode fiber structure," Japanese Journal of Applied Physics, 2018, 57(9): 1-5.

[13] H. F. Du, X. Y. Sun, Y. W. Hu, X. R. Dong, and J. H. Zhou, "High sensitive refractive index sensor based on cladding etched photonic crystal fiber Mach-Zehnder interferometer," Photonic Sensors, 2019, 9(2): 126-134.

[14] X. Y. Sun, X. R. Dong, Y. W. Hu, H. T. Li, D. K. Chu, J. Y. Zhou, et al., "A robust high refractive index sensitivity fiber Mach-Zehnder interferometer fabricated by femtosecond laser machining and chemical etching," Sensors and Actuators A: Physical, 2015, 230: 111-116.
[15] L. V. Nguyen, D. Hwang, S. Moon, D. S. Moon, and Y. Chung, "High temperature fiber sensor with high sensitivity based on core diameter mismatch," Optics Express, 2008, 16(15): 11369-11375.

[16] Y. Ma, X. Q. Qiao, T. A. Guo, R. H. Wang, J. Zhang, Y. Y. Weng, et al., "Mach-Zehnder interferometer based on a sandwich fiber structure for refractive index measurement," IEEE Sensors Journal, 2012, 12(6): 2081-2085.

[17] Y. Zhao, F. Xia, M. Q. Chen, Y. Zhao, F. Xia, and M. Q. Chen, "Curvature sensor based on Mach-Zehnder interferometer with vase-shaped tapers," Sensors and Actuators A: Physical, 2017, 265: 275-279.

[18] L. Mao, P. Lu, Z. Lao, and D. Liu, "In-fiber Mach-Zehnder interferometer based on multi-mode fiber and up-taper for curvature sensing," Optik, 2014, 125(18): 5108-5111.

[19] C. Shen, C. Zhong, H. Gong, J. Wang, J. Chu, $\mathrm{X}$. Zou, et al., "Polarization-dependent curvature sensor based on an in-fiber Mach-Zehnder interferometer with a difference arithmetic demodulation method," Optics Express, 2012, 20(14): 15406-15417.

[20] Y. Gong, T. Zhao, Y. J. Rao, and Y. Wu, "All-fiber curvature sensor based on multimode interference," IEEE Photonics Technology Letters, 2011, 23(11): 679-681.

[21] H. P. Gong, H. F. Song, X. R. Li, J. F. Wang, and X. Y. Dong, "An optical fiber curvature sensor based on photonic crystal fiber modal interferometer," Sensors and Actuators A: Physical, 2013, 195: 139-141.

[22] R. H. Wang, J. Zhang, Y. Y. Weng, Q. Z. Rong, Y. Ma, Z. Y. Feng, et al., "Highly sensitive curvature sensor using an in-fiber Mach-Zehnder interferometer," IEEE Sensors Journal, 2013, 13(5): 1766-1770.

[23] Y. Zhao, L. Cai, and X. G. Li, "Temperatureinsensitive optical fiber curvature sensor based on SMF-MMF-TCSMF-MMF-SMF structure," IEEE Transactions on Instrumentation \& Measurement, 2016, 66(1): 141-147.

[24] H. Sun, S. Yang, J. Zhang, Q. Z. Rong, L, Liang, Q. $\mathrm{F}$. $\mathrm{Xu}$, et al., "Temperature and refractive index sensing characteristics of an MZI-based multimode fiber-dispersion compensation fiber-multimode fiber structure," Optical Fiber Technology, 2012, 18(6): 425-429.

[25] Y. M. Raji, H. S. Lin, S. A. Ibrahim, M. R. Mokhtar, and Z. Yusoff, "Intensity-modulated abrupt tapered fiber Mach-Zehnder interferometer for the simultaneous sensing of temperature and curvature," Optics \& Laser Technology, 2016, 86: 8-13.

[26] X. R. Dong, H. F. Du, X. Y. Sun, and J. A. Duan, "Simultaneous strain and temperature sensor based on a fiber Mach-Zehnder interferometer coated with 
Pt by iron sputtering technology," Materials, 2018, 11(9): 1535-1545.

[27] C. X. Lu, J. A. Su, X. P. Dong, T. Sun, and K. T. V. Grattan, "Simultaneous measurement of strain and temperature with a few-mode fiber-based sensor," Journal of Lightwave Technology, 2018, 36(13): 2796-2802.

[28] C. Li, T. G. Ning, C, Zhang, J. Li, C. B. Zhang, X. D. Wen, et al., "All-fiber multipath Mach-Zehnder interferometer based on a four-core fiber for sensing applications," Sensors and Actuators A: Physical, 2016, 248: 148-154.

[29] L. L. Mao, P. Lu, Z. F. Lao, D. M. Liu, and J. S. Zhang, "Highly sensitive curvature sensor based on single-mode fiber using core-offset splicing," Optics
\& Laser Technology, 2014, 57: 39-43.

[30] Y. Wu, L. Pei, W. X. Jin, Y. C. Jiang, Y. G. Yang, and $\mathrm{Y}$. Shen, "Highly sensitive curvature sensor based on asymmetrical twin core fiber and multimode fiber," Optics \& Laser Technology, 2017, 92: 74-79.

[31] Y. Zhao, C. Lu, and X. Li, "Temperature-insensitive optical fiber curvature sensor based on SMF-MMF-TCSMF-MMF-SMF structure," IEEE Transactions on Instrumentation and Measurement, 2016, 66(1): 141-147.

[32] W. Jin, C. Michie, G. Thursby, M. Konstantaki, and B. Culshaw, "Simultaneous measurement of strain and temperature: error analysis," Optical Engineering, 1997, 36(2): 598-609. 